\title{
PERFIL EPIDEMIOLÓGICO DAS URGÊNCIAS E EMERGÊNCIAS OFTALMOLÓGICAS DO SERTÃO PARAIBANO
}

\author{
EPIDEMIOLOGICAL PROFILE OF THE OPHTHALMOLOGICAL \\ URGENCIES AND EMERGENCIES OF SERTÃO PARAIBANO
}

\author{
Fernanda de Souza Maurício ${ }^{1}$ \\ José Liberlando Alves de Albuquerque ${ }^{2}$ \\ Renata Lívia Silva Fonseca Moreira de Medeiros ${ }^{3}$ \\ Paulo André Guerra Calazans ${ }^{4}$ \\ Ricardo Lourenço Coelho ${ }^{5}$
}

RESUMO: INTRODUÇÃO: As urgências e emergências (UE) oftalmológicas, em destaque os traumas oculares, apresentam maior morbidade, inferindo na qualidade de vida das pessoas acometidas. Outros fatores que interferem na qualidade de vida, são a falta de assistência imediata e o manejo inadequado. Isso tudo leva a um impacto importante no sistema de saúde, tanto privado quanto público, sendo pertinente avaliar a estrutura e os profissionais quanto à capacidade de lidar com tais tipos de afecções e, assim, receber o paciente em cada serviço. É importante ter em mente o direito a uma visão íntegra, a qual impacta diretamente na qualidade da sobrevivência humana, sendo imprescindível estudar o perfil epidemiológico e a inclinação dos traumas em pacientes vítimas de UE oftalmológicas. OBJETIVO: Caracterizar os pacientes que procuraram o centro oftalmológico por terem sido vítimas de urgências e emergências oftalmológicas, identificar o perfil epidemiológico e classificar as principais urgências e emergências. METODOLOGIA: Trata-se de um projeto de pesquisa de campo, documental, analítico observacional, a partir de um estudo de coorte, longitudinal, através da análise prospectiva dos casos, de natureza quantitativa. A pesquisa foi realizada no Centro Médico Sabino Rolim Guimaraes do município de Cajazeiras-PB. RESULTADOS: O sexo masculino

\footnotetext{
1 Discente de Bacharelado em Medicina da Faculdade Santa Maria. E-mail: fernandasmauricio25@gmail.com.

2 Docente da Faculdade Santa Maria. Graduação em Medicina pela Universidade Federal do Ceará, Brasil (1992). E-mail: josealbuquerque.ja@gamil.com.

3 Docente da Faculdade Santa Maria. Doutorado em pesquisa em cirurgia pela faculdade de ciências médica (2018). E-mail: renaliviamoreira@hotmail.com.

${ }^{4}$ Discente de Bacharelado em Medicina da Faculdade Santa Maria. E-mail: calazanspag@gmail.com.

5 Docente da Faculdade Santa Maria. Especialização - residência médica em oftalmologia pelo hospital de olhos Keira de Andrade, Brasil (2001). E-mail: coelhoricardi@ig.com.br.
} 
representou $57 \%$ no total de atendimentos; adultos jovens de 19 até 39 anos, representando $42,8 \%$ da amostra; $O$ grupo dos trabalhadores ativos foi o mais acometido com 62,5\%. CONCLUSÃO: O sexo masculino demonstrou maior vulnerabilidade as urgências e emergências oftalmológicas no serviço especializado. A faixa etária mais acometida foi a de adultos jovens. A ocupação mais frequente se deu pelos trabalhadores ativos na sua profissão.

PALAVRAS CHAVE: Emergências. Perfil epidemiológico. Oftalmológicas.

ABSTRACT: INTRODUCTION: Ophthalmological urgencies and emergencies (UE), especially eye trauma, present higher morbidity, inferring in the quality of life of affected people. Other factors that interfere with the quality of life are the lack of immediate assistance and inadequate management. This all has an important impact on the health system, both private and public, and it is pertinent to evaluate the structure and the professionals as to their ability to deal with these types of conditions and thus receive the patient in each service. It is important to bear in mind the right to a full view, which directly impacts the quality of human survival. It is essential to study the epidemiological profile and the inclination of trauma in patients suffering from ophthalmologic UE. OBJECTIVE: To characterize the patients who sought the ophthalmic center for being victims of ophthalmic emergencies, to identify the epidemiological profile and to classify the main urgencies and emergencies. METHODOLOGY: This is a field research project, documentary, observational analytical, based on a longitudinal cohort study, through the prospective analysis of cases, of quantitative nature. The research was carried out at Sabino Rolim Guimaraes Medical Center of Cajazeiras - PB. RESULTS: Males accounted for $57 \%$ of total consultations; young adults from 19 to 39 years old, representing $42.8 \%$ of the sample; The group of active workers was the most affected with $62.5 \%$. CONCLUSION: The male gender showed greater vulnerability to urgent and ophthalmic emergencies in the specialized service. The most affected age group was young adults. The most frequent occupation was by workers active in their profession.

Descriptors: Emergencies. Epidemiological profile. Ophthalmologic. 


\section{INTRODUÇÃO}

As urgências oftalmológicas, mais comumente os traumas oculares, possuem importante destaque na morbidade e qualidade de vida dos indivíduos acometidos por tais afecções. Impactam diretamente no sistema de saúde, tanto público quanto privado, e urgem pronta resolutividade do quadro clínico, necessária em decorrência dessas afecções serem extremamente incômodas.

Com o objetivo de prevenir qualquer dano irreversível, sendo a visão de imprescindível importância, principalmente para a População Economicamente Ativa (PEA). Dubeux; Freese; Felisberto, em 2013, estimaram que, nos Estados Unidos da América (EUA) ocorram aproximadamente 2,4 milhões traumas oculares por ano. Já dados aferidos no Brasil, demonstram que 10\% dos traumatismos ocupacionais são oculares, Adam Netto et al. (2006).Sendo que, a carência de dados e informações não permite inferir um número aproximado de afecções dessa natureza no Brasil, visto não haver um consolidado banco de dados capaz de fornecer tais informações.

O estudo das Urgências e Emergências (UE) oftalmológicas é relevante por representar graves consequências à saúde, visto que, quando não há a assistência imediata ou por falta do manejo adequado, tem-se um impacto na qualidade de vida dos pacientes acometidos (FERRAZ; MANTOVAN; MELLO, 2016). Sendo pertinente avaliar se as urgências e emergências, tanto do Sistema Único de Saúde (SUS) quanto do sistema privado possuem estrutura e profissionais capazes de lidar com tais tipos de afecções. Ou se existem serviços de referência de pronto atendimento, especialmente nas regiões mais distantes das capitais brasileiras e com menos acesso aos profissionais de saúde.

Estudos realizados por Matos (2018) destacam os corpos estranhos como sendo a principal causa de traumas oculares, com uma incidência que varia de $54,6 \%$ a $81,8 \%$ do total de casos. O diagnóstico dessas urgências é feito por sinais oftalmológicos e sintomas específicos, com auxílio dos exames complementares. 
Portanto, o médico deve saber conduzir adequadamente o seu tratamento, realizando tal procedimento com técnica adequada.

Existem poucas referências assertivas na literatura brasileira que dão ênfase ao trauma ocular, em geral, ignorando certos aspectos epidemiológicos de extrema relevância, os quais entrariam de acordo com a promoção de medidas educacionais preventivas e direcionamento para o serviço especializado.

Deve-se salientar que o direito a uma visão íntegra, requer uma premissa para a qualidade da sobrevivência humana, como condição necessária para executar qualquer atividade, portanto, é imprescindível estudar o perfil epidemiológico e a inclinação dos traumas em pacientes vítimas de UE oftalmológicas, haja vista que esse tipo de conhecimento, ao longo dos anos, permite apresentar e revisar estratégias de prevenção. Assim, este estudo tem por objetivo analisar as frequência das UE oftalmológicas em um serviço particular do interior paraibano, aferindo a ocorrência por gênero, faixa etária, acidentes ocupacionais, dentre outras causas de afecções oculares. Procurando estabelecer um entendimento sobre a necessidade de realizar medidas preventivas e como estas deveriam ser implementadas.

\section{MATERIAIS E MÉTODOS}

O estudo foi realizado a partir da análise prospectiva, longitudinal, documental, de natureza quantitativa, através de um instrumento de coleta de dados, preenchidos com informações dos prontuários dos pacientes atendidos em caráter emergencial no ambulatório de oftalmologia de um serviço particular do sertão paraibano, coletados no período do mês de novembro do ano de 2018 ao mês de fevereiro de 2019.

A coleta dos dados foi realizada após anuência do Comitê de Ética em Pesquisa da Faculdade Santa Maria, em conformidade com a resolução 466/12 do Conselho Nacional de Saúde, e com o consentimento do uso de dados pela direção 
do serviço, seguindo os parâmetros éticos e preocupando-se em obedecer a integridade e anonimato de identidade da população analisada.

Foram 112 (cento e doze) pacientes atendidos pelo serviço de urgência/emergência do ambulatório de oftalmologia, quase enquadraram nos critérios de inclusão estabelecidos pelo estudo (idade superior a 18 anos, sem consulta pré-agendada, prontuários de pacientes registrados pelo estabelecimento como consulta de UE).

A partir da análise dos prontuários, com o devido preenchimento do instrumento próprio para a coleta das informações referentes ao trauma ocorrido e à caracterização sócio demográfica dos pacientes, foi possível realizar um compilado foi utilizado um instrumento elaborado a partir de questões referentes à caracterização sociodemográfica dos pacientes.

\section{RESULTADOS}

A partir da análise dos prontuários utilizados, foi possível realizar uma análise descritiva do perfil sócio demográfico da amostra. Quanto ao gênero, o sexo masculino foi ode maior frequência, representando $57 \%$ (64) da amostra, sendo o sexo feminino apenas $43 \%$ (48) da amostra, de acordo com o gráfico 1. 
Gráfico 1: Distribuição dos pacientes de acordo com o gênero.

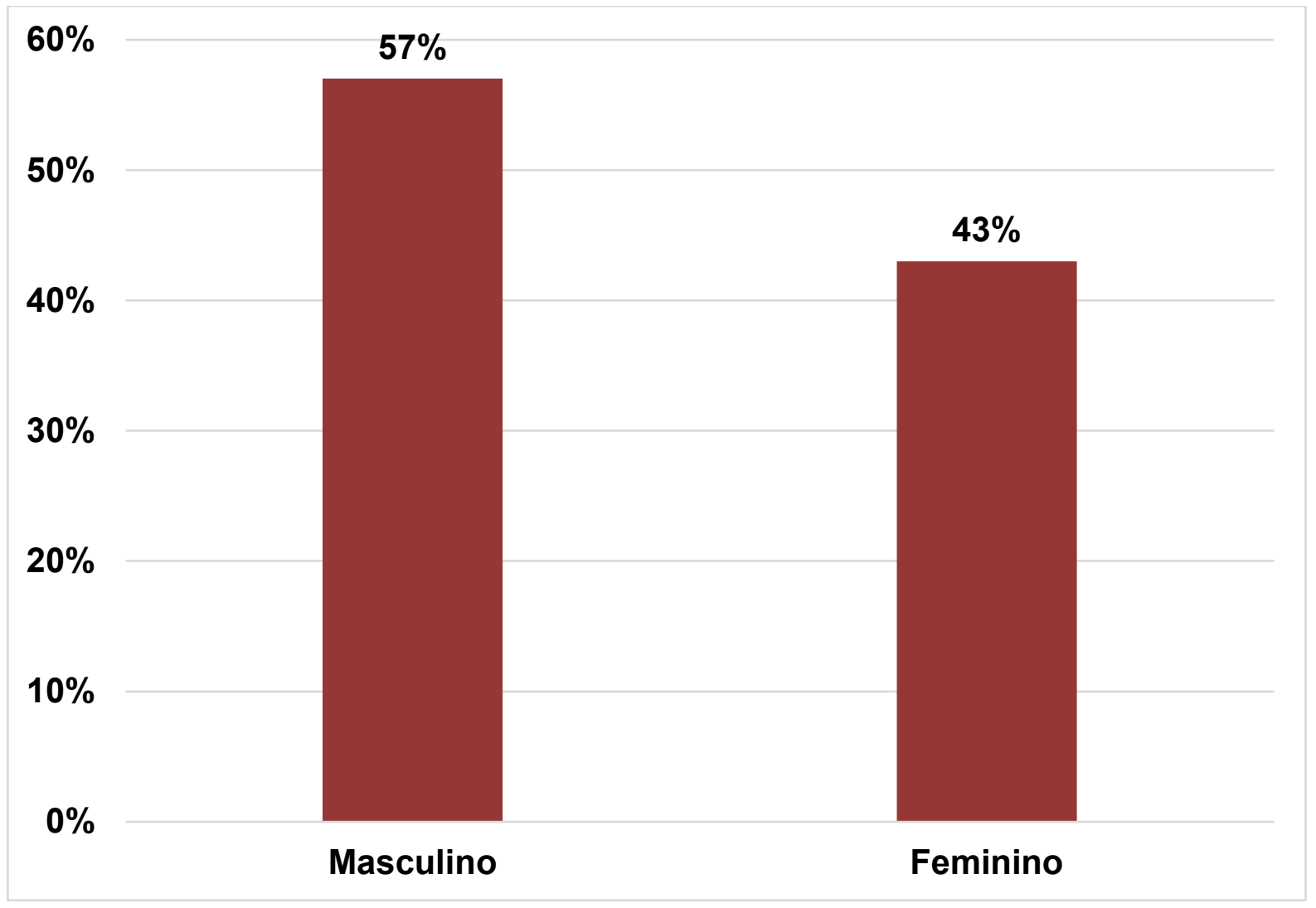

Fonte: dados da pesquisa, 2019.

A idade mínima e máxima variou entre 19 e 93 anos, obtendo-se predomínio entre os adultos jovens de 19 até 39 anos, representando 42,8\% da amostra. Faixa etária de idosos, idade superior a 60 anos, foi representada por apenas $31,3 \%$ (35) da amostra, sendo seguido por 25,9\% representados por adultos com idade entre 40 à 59 anos, como demonstrado pelo (Gráfico 2). 
Gráfico 2: Distribuição dos pacientes de acordo com a faixas etárias.

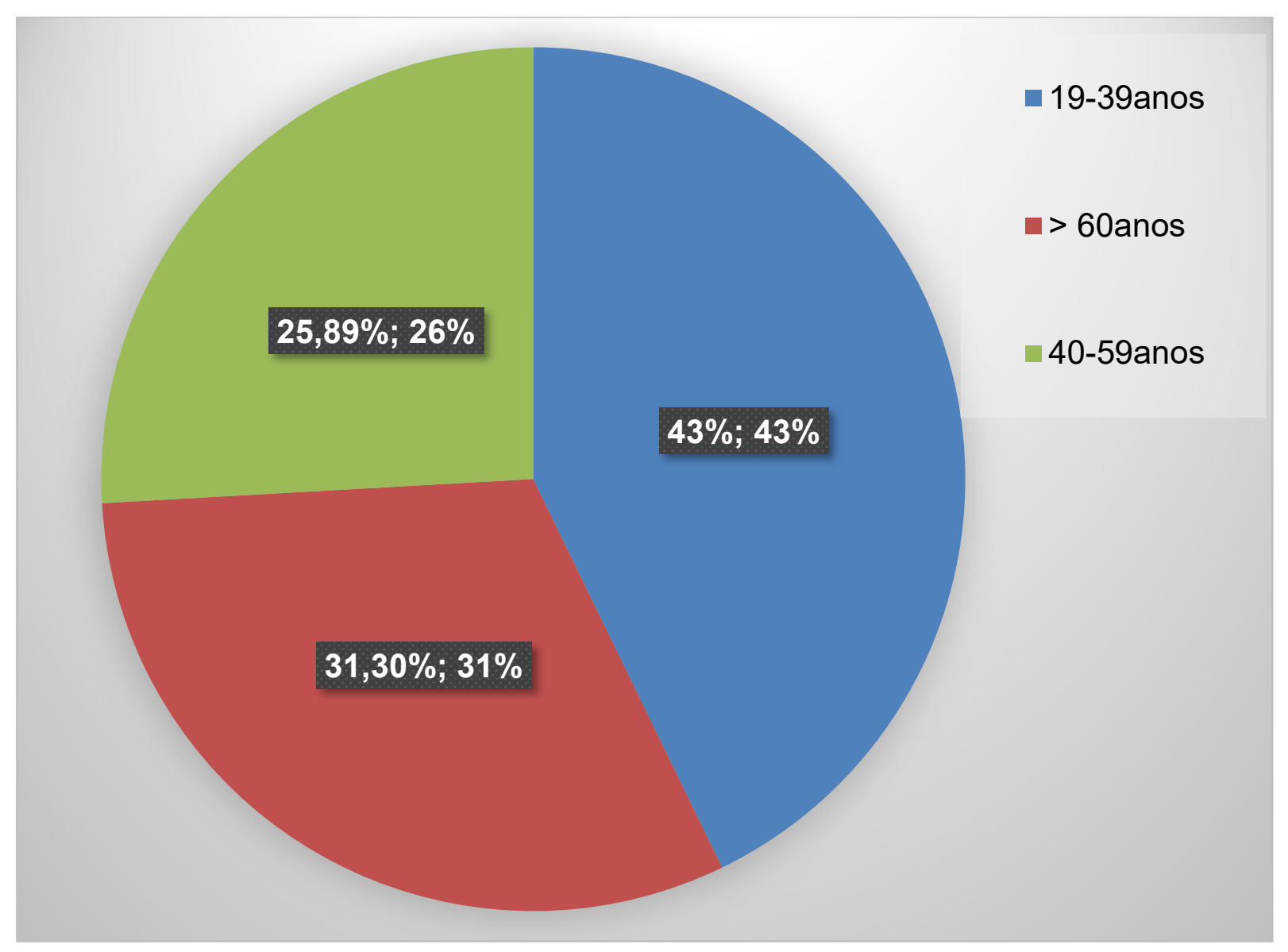

Fonte: dados da pesquisa, 2019.

Para a análise da relação e avaliação da frequência entre UE oftalmológicas e a PEA, a amostra foi classificada em grupos: 1. População Economicamente Ativa; 2. Estudantes; 3. Aposentados; 4. Do lar. O grupo dos trabalhadores ativos foi o mais acometido com $62,5 \%$ (70) dos casos. Em seguida, os aposentados representados por $24,1 \%$ e pessoas do lar, com $1,8 \%$, como pode ser avaliado pelo gráfico 3. 
Gráfico 3: Avaliação da frequência entre UE oftalmológicas e a PEA.

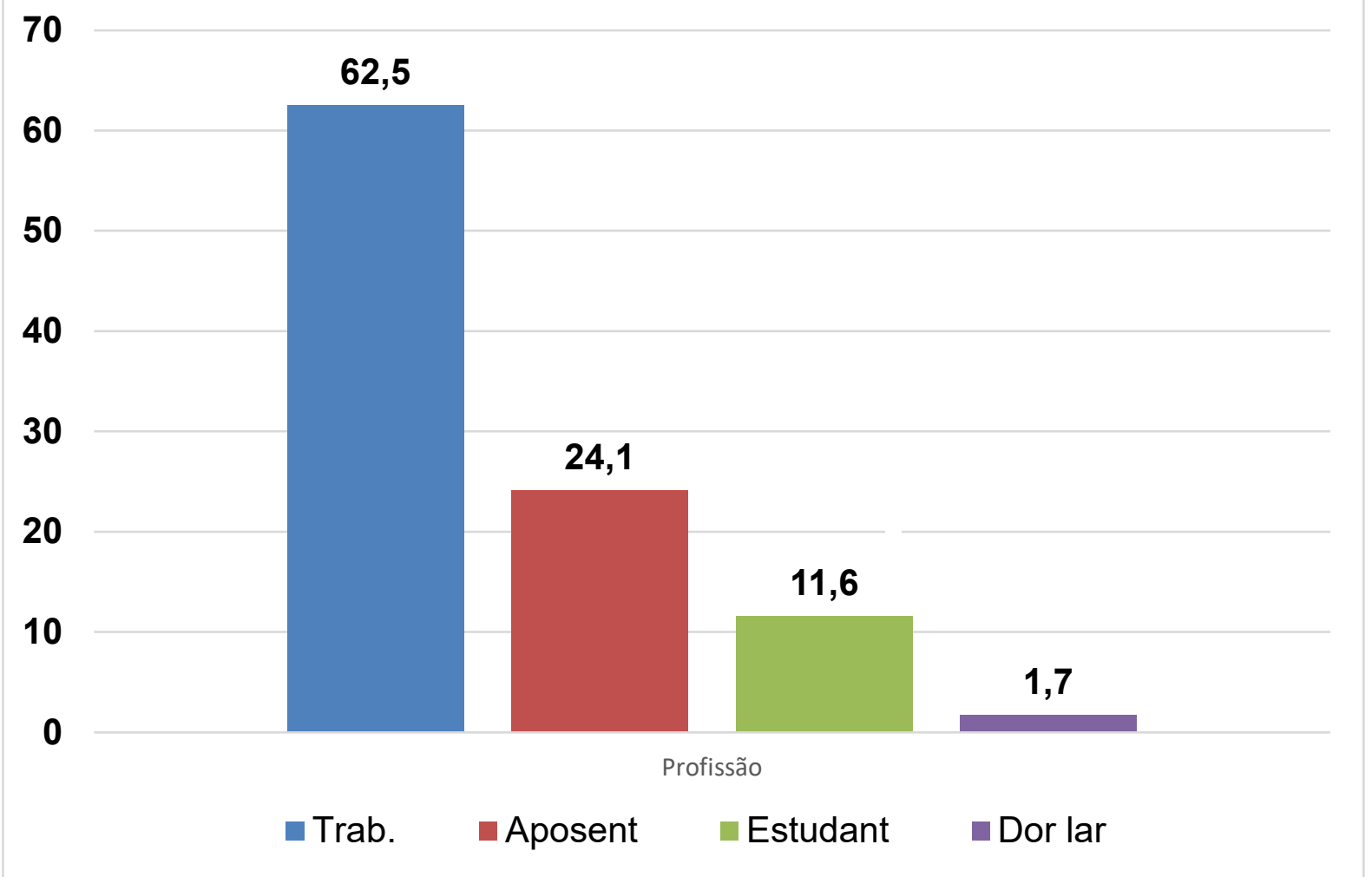

Fonte: dados da pesquisa, 2019.

\section{DISCUSSÃO}

A partir dos resultados obtidos, foi possível avaliar a notoriedade da predominância do sexo masculino como o gênero mais acometido por urgências e/ou emergências oculares, sendo consonante tal achado com estudos publicados por Adam Netto et al. (2006). Pode-se inferir que tal achado deve-se ao fato de maior risco ocupacional dos homens as atividades com alta periculosidade, mesmo portando os Equipamentos de Proteção Individual (EPI) necessários para tais atividades, ou ainda ao fato de homens, principalmente os mais jovens, estarem mais predispostos a lesões por causas externas, por imprudência e/ou negligência com os EPIs necessários para suas atividades laborais, como afirma o estudo realizado por Cisz (2015). 
A faixa etária com maior prevalência é de adultos jovens, com idade entre 19 e 39anos, ratificando que os homens adultos jovens e ativos no mercado de trabalho são mais susceptíveis a acidentes oftalmológicos, tanto por estarem expostos aos riscos pela rotina diária, seja pela falta de orientação do uso correto dos equipamentos de segurança, ou também possivelmente pela decorrência de condições inapropriadas no ambiente de trabalho. Segundo Almeida; Pagliuca; Leite (2005) esses acidente de trabalho são identificados caracterizando-se por um contato direto, abrupto e impensado entre a pessoa e o agente agressor em um momento não preestabelecido, tomando como inesperado. Esse tipo de acidente está diretamente interligado aos riscos ocupacionais, ou seja, aos componentes existentes no local de trabalho, podendo ser nocivos à saúde do profissional, gerando ao decorrer do tempo, doenças ocupacionais adquiridas.

Segundo a Organização Pan-Americana de Saúde, os cuidados iniciais com a saúde dos olhos devem ser direcionados para aqueles mais expostos a riscos, como crianças, idosos e pessoas cujo trabalho envolve exposição do sistema visual. Confirmando com o resultado do presente estudo, que demonstrou que adultosjovens, os quais possuem atuação profissional ativa são mais expostos a riscos por sua exposição ocupacional. Mostrando a necessidade de maior incentivo aos cuidados pré-exposição ao ambiente de trabalho.

A higiene do trabalho envolve o estudo e controle das condições de trabalho que influenciarão diretamente na sua qualidade, na saúde física e bem-estar do trabalhador no seu local de trabalho.

Ramos (2009) evidencia que os óculos de proteção, importante EPI, são imprescindíveis para que não ocorra uma perfuração do globo ocular por meio de corpos estranhos. Ademais, é visto em estudos que o fato de não usar ou negligenciar o uso adequado e apropriado de EPIs é um dos fatores que mais contribuiu para os acidentes no ambiente de trabalho pesquisado.

Cerca de 70\% dos indivíduos pesquisados por Cisz (2015) já foram submetidos por algum acidente de trabalho pelo não uso correto dos EPIS, admitindo que a causa desse tipo de trauma foi devido ao não uso, ou ao uso incorreto, dos EPI's. Ainda de acordo com esse mesmo autor, a conscientização do uso de EPI'S, e sua devida importância deve ser realizada pela Comissão Interna de 
Prevenção de Acidentes (CIPA), responsável quanto à segurança pessoal e coletiva. A CIPA tem como objetivo sugerir medidas de prevenção de acidentes por iniciativa própria ou sugestões, encaminhando-os ao empregador; promover a divulgação e zelar pela observância das normas de segurança e medicina do trabalho e promover, anualmente, a Semana Interna de Prevenção de Acidentes de Trabalho SIPAT. Portanto, diante dessas atribuições, é necessária a execução dessas medidas no ambiente hospitalar para, com isso, prevenir e reduzir esses tão elevados índices de acidentes.

Estudos apresentam que em torno de $50 \%$ dos pacientes que buscam serviço de urgência oftalmológica apresentam história de trauma. No presente estudo os casos de emergência oculares, incluindo trauma, queimaduras, corpo estranho, irritação, representaram 48\% dos atendimentos (PEREIRA et al.,2011).

É total acessão em estudos que a ocorrência de traumas e outras emergências oculares entre a população vista neste estudo poderia ser eficientemente reduzida com o treinamento e incentivo ao uso rotineiro de EPIs.

Portanto, em relação ao grupo de pacientes vítimas emergências oculares já citado, é visto a necessidade de informações por parte das instituições governamentais e trabalhistas quanto à prevenção de acidentes ocupacionais, ademais da orientação coerente a estes adultos jovens ativos e ao uso de equipamentos de proteção individual.

Segundo Gómes; Caballero-Palomares (2002), um quinto dos adultos jovens sofre trauma ocular durante sua vida, representando uma das causas mais importantes de cegueira e deficiência visual. Foi relatado que $16 \%$ dos pacientes com trauma grave e 55\% dos pacientes com trauma facial apresentam trauma ocular ou orbital e $20 \%$ dos pacientes com trauma facial perde a visão em um olho. Traumatismos mais comumente relatados em serviços de UE são hiperemia conjuntival, abrasões da córnea e hemorragia subconjuntival. O primeiro atendimento imediato desses pacientes, segundo ATLS (suporte avançado de vida em trauma) o qual entra na avaliação secundária, traz como proposta o exame oftalmológico para procurar intencionalmente um grupo de lesões consideradas mais graves ou mais frequentes nas diferentes estruturas oculares, para assim, evitar sequelas irreversíveis. 
Segundo Santana et al. (2006) os acidentes de trabalho causam um grande impacto sobre a produtividade e a economia, além de grande sofrimento para a sociedade, podendo ser evitados, de modo a eliminar todos esses transtornos. Segundo a Organização Internacional do Trabalho (OIT), ocorrem cerca de 270 milhões de acidentes de trabalho e cerca de dois milhões de mortes por ano em todo o mundo que, sendo possivelmente preveníveis, demonstram abandono e desigualdade social.

Ainda de acordo com os autores, é visto que no Brasil, no ano de 2002 , baseado no Produto Interno Bruto (PIB), foram realizadas estimativas, resultando em perdas financeiras altamente significativas, sendo reflexo de uma baixa efetividade das políticas e programas de prevenção de agravos à saúde no trabalho. Estudos sobre essas estimativas dos custos dos acidentes de trabalho vêm aumento ano após ano. Esses gastos incluem os diretos, relacionados as despesas médicas, como tratamento e reabilitação, e as não médicas, como o transporte, que representara $34 \%$ do total. Os custos indiretos, são representados pela perda da produtividade e da produção, indenizações e compensações salariais, sendo estimados em US\$96.2 bilhões.

Vilela, Almeida, Mendes (2012) afirmam que alguns Centro de Referência em Saúde do Trabalhador (CEREST), incluem a academia em projeto conjunto de pesquisa em políticas públicas, com objetivo de tornar viável o Sistema de Vigilância de Acidentes de Trabalho (SIVAT), que consiste em notificar os acontecimentos nas unidades de urgência, realizando o processamento dos dados e intervenção realizadas nos casos graves e fatais, ou nas empresas com maior proporção de incidência. Nos últimos três anos, após 2006, realizou-se o desenvolvimento do segundo projeto de pesquisa com apoio da Fundação de Amparo à Pesquisa do Estado de São Paulo (FAPESP), tendo como objeto aprimorar o SIVAT. Esse aprimoramento teve como objeto ações sistemáticas de vigilância, para estudar os motivos que influem na não redução desses índices. 


\section{CONCLUSÕES}

A partir dos resultados obtidos, foi observado a prevalência do sexo masculino em urgências/emergências oftalmológicas no serviço especializado. Predominância da faixa etária de adultos jovens que se encontram economicamente ativos. Podendo-se inferir que tais resultados sejam em decorrência de acidentes ocupacionais.

\section{REFERÊNCIAS BIBLIOGRÁFICAS}

ALMEIDA, C.B.; PAGLIUCA, L.M.F.; LEITE, A.L.A.S. Acidentes de trabalho envolvendo os olhos: avaliação de riscos ocupacionais com trabalhadores de enfermagem. Rev. Latino-Am. Enfermagem, Ribeirão Preto, v.13, n. 5, p. 708-716.

ADAM NETTO, A. Perfil epidemiológico de 144 pacientes portadores de corpos estranhos subtarsais no Hospital Universitário da Universidade Federal de Santa Catarina. Arquivos Catarinenses de Medicina. Vol. 35, N. 4, 2006, p. 97-103.

CISZ, C.R. Conscientização do uso de EPI'S, quanto à segurança pessoal e coletiva. Monografia para o curso de Especialização em Engenharia de Segurança do Trabalho. Paraná: Universidade Tecnológica Federal do Paraná, 2015.

DUBEUX, L.S.; FREESE, E.; FELISBERTO, E. Acesso a hospitais regionais de urgência e emergência: abordagem aos usuários para avaliação do itinerário e dos obstáculos aos serviços de saúde. Physis, Rio de Janeiro, v. 23, n. 2, p. 345-369, June 2013.

FERRAZ, R.M.; MANTOVAN, F.B.; MELLO, E.M. Relação entre queixa e diagnóstico no pronto socorro de oftalmologia. Colloq Vitae, V.8, N.3, 2016, p.: 60-67.

GÓMEZ, V.L.; CABALLERO-PALOMARES, M. Trauma ocular: distribución de acuerdo con la clasificación estandardizada. Trauma, Vol. 5, Núm. 1, 2002, pp 5-10.

MATOS, A. G. Rotinas em emergências oftalmológicas. Fortaleza-ceará: Cultura Médica, 2018.

PEREIRA, F.B. et al. Perfil da demanda e morbidade dos pacientes atendidos em centro de urgências oftalmológicas de um hospital universitário. Rev. Bras. Oftalmol. V.70, n.4, 2011, p.: $238-42$.

RAMOS, P. Análise do Programa de Prevenção de Acidentes - Quase Acidente - e a Viabilidade da Aplicação Direta na Construção Civil - Estudo de Caso. Trabalho e Conclusão de Curso submetido à Universidade do Extremo Sul Catarinense - UNESC - no ano de 2009. 
SANTANA, V.S.; et al. Acidentes de trabalho: custos previdenciários e dias de trabalho perdido. Rev. Saúde Pública. V.40, N.6, p.1004-12.

VILELA, R.A.G.; ALMEIDA, I.M.; MENDES, V.A. Da vigilância para prevenção de acidentes de trabalho: contribuição da ergonomia da atividade. Ciência \& Saúde Coletiva, v. 17, n. 10, p.: 2817-2830. 